\title{
HST observations of the metal rich globular clusters NGC 6496 and NGC 6352^
}

\author{
L. Pulone ${ }^{1}$, G. De Marchi ${ }^{2}$, S. Covino ${ }^{3}$, and F. Paresce ${ }^{4}$ \\ 1 INAF, Osservatorio Astronomico di Roma, Via di Frascati 33, 00040 Monte Porzio Catone (RM), Italy \\ 2 European Space Agency, Space Telescope Operations Division, 3700 San Martin Drive, Baltimore MD 21218, USA \\ 3 INAF, Osservatorio Astronomico di Brera, Via Bianchi 46, 23807 Merate (LC), Italy \\ ${ }^{4}$ European Southern Observatory, Karl-Schwarzschild-Strasse 2, 85748, Garching, Germany
}

Received 12 June 2002 / Accepted 27 November 2002

\begin{abstract}
Deep exposures of the metal-rich globular clusters NGC 6496 and NGC 6352 were obtained with the WFPC2 camera on board the Hubble Space Telescope (HST) through the F606W and F814W filters. The resulting colour-magnitude diagrams (CMD) reach down to absolute magnitude $M_{814} \simeq 10-10.5$, approximately 5 magnitudes below the main sequence (MS) turnoff (TO). The MS of the two clusters are sharp and well defined and their fiducial lines overlap almost exactly throughout this range. Their colour is, however, more than 0.1 mag redder than the MS fiducial line of the prototype metal-rich globular cluster 47 Tuc (NGC 104), after proper correction for the relative distances and reddening. This provides solid empirical evidence of a higher metal content, which is not surprising if these objects belong indeed to the bulge as their present location suggests. A good fit to the upper part of the MS of both clusters is obtained with a $10 \mathrm{Gyr}$-old theoretical isochrone from Baraffe et al. (1998) for a metallicity of $[\mathrm{M} / \mathrm{H}]=-0.5$, but at lower luminosities all models depart considerably from the observations, probably because of a deficiency in the treatment of the TiO opacity. The luminosity functions (LF) obtained from the observed CMD are rather similar to one another and show a peak at $M_{814} \simeq 9$. The present day mass functions (PDMF) of both clusters are derived down to $M_{814} \simeq 10.5$ or $m \simeq 0.2 M_{\odot}$ and are consistent with power-law indices $\alpha=0.7$ for NGC 6496 and $\alpha=0.6$ for NGC 6352. The PDMF of NGC 104 is twice as steep in the same mass range $(\alpha=1.4)$. We investigate the origin of this discrepancy and show that it can be understood if the two clusters contain a considerably higher fraction of primordial binaries amongst their MS population, similar to that expected in the bulge. We briefly discuss the implications of this finding on the process of star and binary formation and on the universality of the IMF.
\end{abstract}

Key words. stars: Hertzsprung-Russell (HR) and C-M diagrams - stars: luminosity function, mass function Galaxy: globular clusters: individual: NGC 6496, NGC 6352

\section{Introduction}

Since the work of Zinn \& West (1984), Armandroff \& Zinn (1988) and Armandroff (1989), the disc population of the Galactic globular clusters (GC) has been recognised as a different subsystem, both dynamically and chemically, compared with that of the halo clusters. A detailed knowledge of these subsystems is important for our understanding of the fundamental properties of GC such as the concentration parameter, the shape of the mass function (MF), their age and chemical composition, since they correlate with the Galactocentric distance (Meylan \& Heggie 1997) and/or height above the Galactic plane. Studying the disc GC, although difficult because of the foreground star contamination and high extinction due to their low Galactic latitude, could shed light on the disruption mechanisms by dynamical friction and bulge shocks,

Send offprint requests to: L. Pulone,

e-mail: pulone@coma.mporzio.astro.it

* Based on observations with the NASA/ESA Hubble Space Telescope, obtained at the Space Telescope Science Institute, which is operated by AURA for NASA under contract NAS5-26555. which deeply modify their MF in time. Furthermore, investigating the physical differences between their stellar population and that of the halo clusters could improve our understanding of the formation process of our Galaxy. In this paper, we present the analysis of the deep CMD of NGC 6496 and NGC 6352, two clusters in the direction of the bulge and putative members of this subgroup, based on observations collected with the WFPC2 on board the HST.

NGC 6496 was originally believed to be a member of the disc system of GC, but Richtler et al. (1994) questioned this classification. They suggested instead that NGC 6496, together with two other clusters, NGC 6624 and NGC 6637, could be halo clusters with strongly inclined orbits. NGC 6496 lies in the Southern sky at $\alpha_{2000}=17^{\circ} 59^{\prime} 02^{\prime \prime}$ and $\delta_{2000}=-44^{\circ} 15^{\prime} 54^{\prime \prime}$. Its Galactic coordinates are $l=348.02^{\circ}$ and $b=-10.1^{\circ}$. A distance of $R_{\mathrm{G}}=4.3 \mathrm{kpc}$ from the Galactic centre and $Z_{\mathrm{G}}=$ $-2.0 \mathrm{kpc}$ below the Galactic plane (Harris 1996), place this object at the edge of the bulge. Notwithstanding the fact that the surrounding field shows no strong stellar foreground variation, its low Galactic latitude has hampered the observation of this cluster from the ground. Indeed, most of the contradictory 
estimates as to its metallicity, reddening, and Galactocentric distance probably arise mainly from the difficulty of observing objects through the dusty and crowded Galactic disc.

Armandroff (1988) presented the first CMD of NGC 6496 in which the photometry reached $\sim 2$ mag below the horizontal branch (HB), disclosing for the first time the usual red arm of the metal-rich clusters. The extinction towards NGC 6496 is uncertain, with estimates ranging between $E(B-V)=0.09$ and $E(B-V)=0.24$ (Armandroff 1988; Zinn \& West 1984; Burstein \& Heiles 1982; Richtler et al. 1994).

The metal content of NGC 6496 has also been controversial for a long time. Friel \& Geisler (1991), in their work on the disc GC based on Washington photometry, used the reddening value of 0.19 by Armandroff (1988) and derived for NGC 6496 a metallicity of $[\mathrm{Fe} / \mathrm{H}]=-1.05$. Richtler et al. (1994) measured its metallicity on the basis of the morphological characteristics of the $\mathrm{CMD}$, obtaining $[\mathrm{Fe} / \mathrm{H}]=-1.0$. Their value is in agreement with the metallicity coming from Washington photometry, but substantially lower than that given by Zinn \& West (1984), i.e. $[\mathrm{Fe} / \mathrm{H}]=-0.48$, and adopted by Armandroff (1988). The discrepancies in the metallicity scale amongst different authors might be explained by the intimate connection between reddening and metallicity estimates.

NGC 6352 is located at $\alpha_{2000}=17^{\circ} 25^{\prime} 29^{\prime \prime}$ and $\delta_{2000}=$ $-48^{\circ} 25^{\prime} 22^{\prime \prime}$. Its Galactic coordinates $\left(l=341.4^{\circ}, b=-7.2^{\circ}\right)$ and distance from the Galactic centre and plane $\left(R_{\mathrm{G}}=3.3 \mathrm{kpc}\right.$, $Z_{\mathrm{G}}=-0.7 \mathrm{kpc}$; Harris 1996) place this objects at the edge or within the bulge. Based on this information and on radial velocity and metallicity measurements, NGC 6352 has been recognised, since Zinn (1985) and Armandroff (1989), as an unambiguous member of the disc group of globular clusters. Sarajedini \& Norris (1994) presented CCD ground-based photometry for NGC 6352 reaching $\sim 2$ mag below the level of its $\mathrm{HB}$, disclosing the red clumped morphology typical of metal-rich clusters. By simultaneous estimate of both metallicity and reddening, using theoretical stellar evolutionary tracks, they obtain $[\mathrm{Fe} / \mathrm{H}]=-0.51$, in the Zinn \& West metallicity scale, and $E(B-V)=0.24$.

Fullton et al. (1995) observed NGC 6352 with the WFPC1 on board HST, obtaining a CMD reaching 3 mag below the MS TO. Their main results are that NGC 6352 is slightly more metal rich than 47 Tuc and that it has the same age as 47 Tuc within the errors of their photometry. These results imply that the primordial gas might have collapsed in a disc configuration at an early epoch of the formation of our Galaxy.

In light of all the uncertainties affecting the determination of the extinction, distance and metallicity of these clusters, the skeptical reader might wonder as to how meaningful and practically useful it is to classify these objects as being members of one or another subgroup of the GC. We believe that the availability of new, powerful instruments such as the HST and VLT should call for a critical re-evaluation of the semi empirical methods on which such classifications were based. In the following sections we provide solid observational data in the form of high precision CMD of NGC 6496 and NGC 6352, covering almost the full range of stellar masses from the TO to $\sim 0.2 M_{\odot}$. We compare them with the CMD of the archetype, well-studied metal-rich globular cluster 47 Tuc (NGC 104) and address the
Table 1. HST observations of the clusters NGC 6496 and NGC 6352.

\begin{tabular}{cccrc}
\hline \hline Cluster & Date UT & Filter & Exp. time (s) & Nr. exp. \\
\hline NGC 6496 & 1999 Apr 1 & F606W & 30 & 1 \\
& & F606W & 40 & 1 \\
& & F606W & 1100 & 2 \\
& & F606W & 1300 & 4 \\
& & F814W & 30 & 1 \\
& & F814W & 40 & 1 \\
NGC 6352 & 1998 Mar 3 & F606W & 1100 & 2 \\
& & F606W & 400 & 4 \\
& & F606W & 1300 & 8 \\
& & F814W & 30 & 3 \\
& & F814W & 40 & 1 \\
& & F814W & 1200 & 2 \\
& & F814W & 1300 & 6 \\
\hline
\end{tabular}

issue of their metallicity in a way so far never attempted, yet robust in light of its simplicity. Furthermore, from the LF we derive the best fitting underlying PDMF to test the hypothesis as to whether the IMF could be metal dependent and, therefore, differ here from that of the metal-poor halo clusters studied by Paresce \& De Marchi (2000).

\section{Observations and data analysis}

All the exposures of both clusters NGC 6496 and NGC 6352, as summarised in Table 1, have been taken in fine lock mode, with the WFPC2 camera on board the HST, through the F606W and F814W broad-band filters.

The images of NGC 6496 were obtained by targetting a region located $\sim 1.3^{\prime}$ East of the cluster centre, at coordinates $\alpha_{2000}=17^{\circ} 59^{\prime} 12^{\prime \prime}$ and $\delta_{2000}=-44^{\circ} 15^{\prime} 59^{\prime \prime}$. The field imaged in our programme is close to the half-light radius $\left(r_{\mathrm{h}}=1.9\right.$; Harris 1996), where the local MF is expected to be less affected by mass segregation resulting from internal dynamical evolution (Richer et al. 1991).

The field observed in NGC 6352 is located at $\alpha_{2000}=$ $17^{\circ} 25^{\prime} 29.2^{\prime \prime}$ and $\delta_{2000}=-48^{\circ} 25^{\prime} 20.2^{\prime \prime}$, or about $2^{\prime}$ North of the cluster centre, at a distance corresponding to the half-light radius ( $r_{\mathrm{h}}=2$ '.0; Harris 1996). The mosaic images of the fields observed in NGC 6496 and NGC 6352 are shown in Figs. 1 and 2, respectively, and are based on two $40 \mathrm{~s}$ F814W exposures (see Table 1).

The raw images were processed using the standard HST pipeline calibration. The long exposures taken through the same filter were combined to remove cosmic ray hits and to improve the signal-to-noise ratio of the data. The total equivalent exposure time of the combined images of NGC 6496 corresponds to $7400 \mathrm{~s}$ in each band. As regards NGC 6352, the total exposure time equals $10400 \mathrm{~s}$ in the F606W band, and $10200 \mathrm{~s}$ in $\mathrm{F} 814 \mathrm{~W}$. The same procedure has been applied to the short exposures, thus producing two $70 \mathrm{~s}$ images in both bands for NGC 6496 and two $130 \mathrm{~s}$ images in both bands for NGC 6352.

Due to the large number of pixel affected by cosmic rays, satellite tracks and detector defects that affected the raw frames 


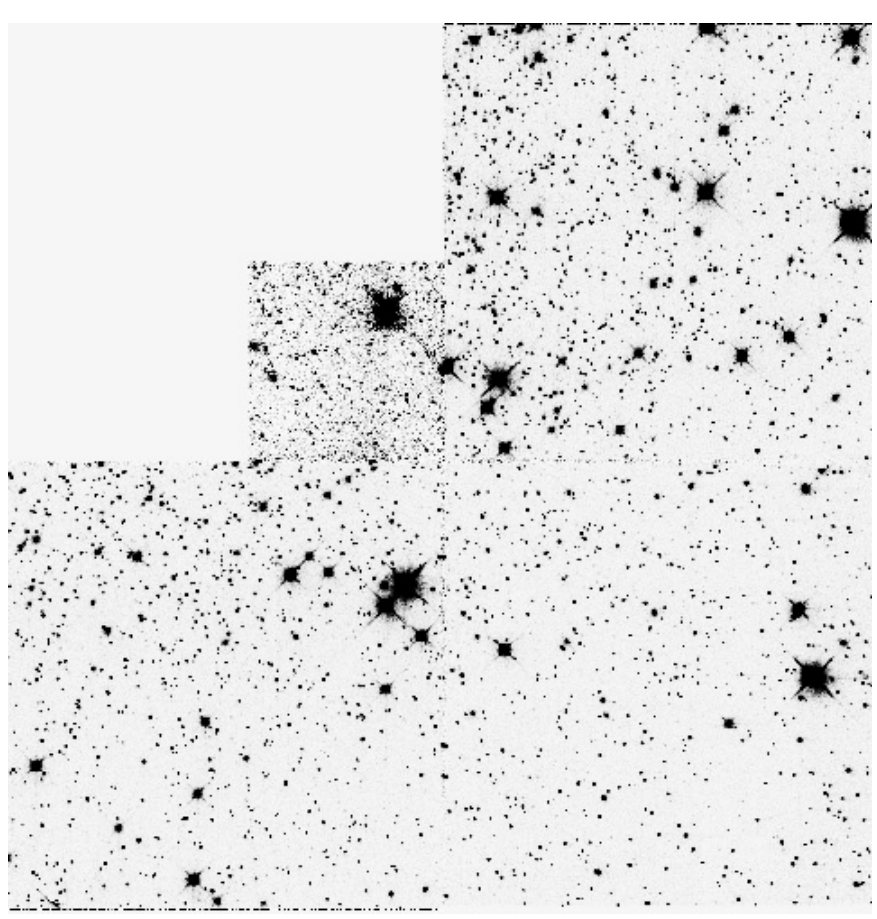

Fig. 1. Negative image of the cluster NGC 6496 of $40 \mathrm{~s}$ duration in the F814W band.

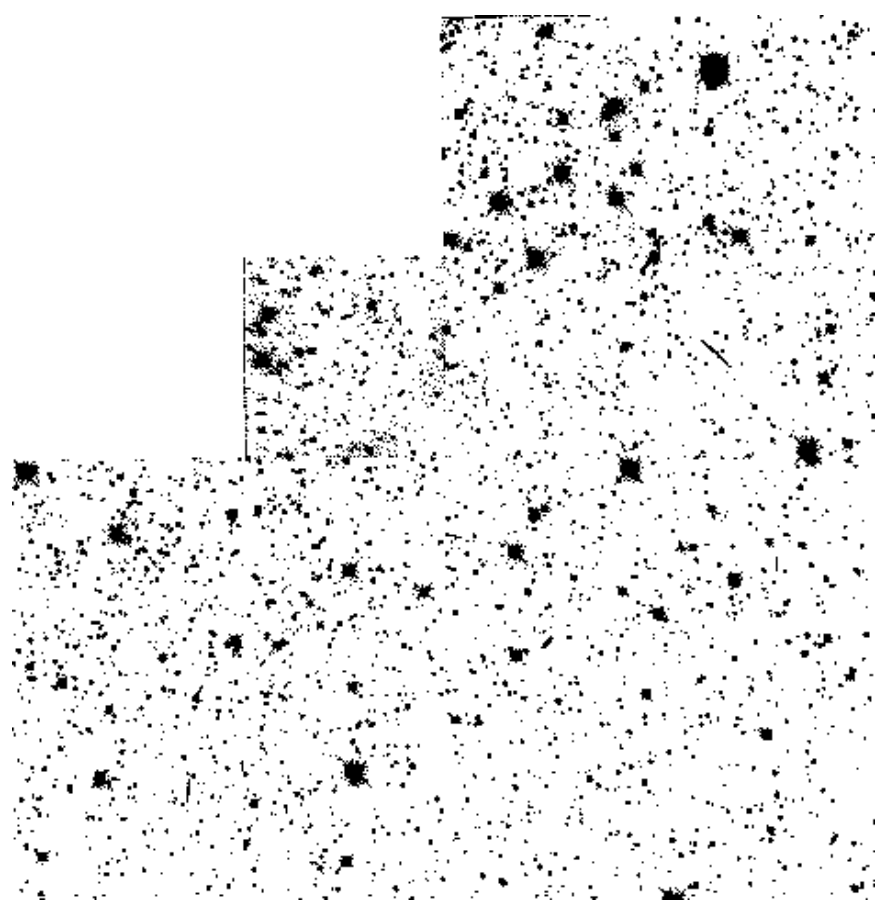

Fig. 2. Negative image of the cluster NGC 6352 of $40 \mathrm{~s}$ duration in the F814W band.

of NGC 6352, however, we decided to derive the final scientific frames for these observations by taking their median rather than the usual mean. As is well known (see for instance the DAOPHOT users' manual; Stetson 1987), the median is very effective in removing outliers, but from a statistical point of view the median of $n$ frames is roughly comparable to the mean of only $2 / 3$ of the frames. Therefore, as far as the star detection algorithm is concerned, our effective exposure time was reduced to $6930 \mathrm{~s}$ and $6800 \mathrm{~s}$ in F606W and F814W, respectively, compared to the ones obtained simply by summing all available frames. The ensuing reduction in the SNR is more than compensated by the higher accuracy achieved in the photometry, resulting in a cleaner CMD and tighter MS.

Photometry was carried out using the standard IRAF DAOPHOT package. The automated star detection routine daophot.daofind was applied to the averaged frames, by setting the detection threshold at $5 \sigma$ above the local background level. We have then visually examined all the detected objects in this way. False identifications, due essentially to the diffraction spikes of a number of saturated stars, were removed from the output list. In this way, concerning the long exposures data set, in the NGC 6496 field a total of 650, 2070, 1500, 2300 objects were detected, respectively in the $P C, W F 2, W F 3$, and WF4 chips. As regards NGC 6352, the detected objects were $379,1443,1279$, and 1216 .

The photometric routines phot and allstar were used to measure the fluxes on the combined frames. The PSF was modelled by averaging moderately bright stars. We calculated the aperture corrections for the four chips, by using a diameter of $0.5^{\prime \prime}$ and translated the final instrumental magnitudes into the VEGAMAG photometric system by adopting the updated zero points listed in the March 1998 edition of the HST Data Handbook. It should be noted that, thanks to the low level of crowding in these images, aperture photometry would have provided equally robust results.

\section{The colour-magnitude diagram}

Figure 3 shows the $m_{814}, m_{606}-m_{814}$ CMD of NGC 6496 and NGC 6352, for all the objects measured in the combined short and long exposures. The photometry reaches $m_{814} \sim 25.5$ and the MS of the clusters are sharp, well defined and stand out clearly from the background contamination down to $m_{814} \sim 24$.

In the long exposures, saturation occurs at $m_{814}<19.5$ in the WF chips and at about one magnitude brighter for the PC (thanks to the higher plate-scale of this camera). Above these brightness limits, the data in Fig. 3 come therefore from the short exposures. The MS changes slope at $m_{814} \simeq 23$ for NGC 6496 and $m_{814} \simeq 22$ for NGC 6352, where it becomes slightly steeper. The change of slope of the MS is due to the effect of dissociation of $\mathrm{H}_{2}$ molecules in the envelope of the star, and to the consequent lowering of the adiabatic gradient (Copeland et al. 1970). This has already been extensively discussed by D'Antona (1995) and D’Antona \& Mazzitelli (1996).

Although the F606W and F814W filters are not the ideal choice for estimating the age of the clusters, the area around their TO is well reproduced by a 14 Gyr old isochrone by Girardi et al. (2002) for a metallicity of $Z=0.008$ and Helium content $Y=0.25$ (see Fig. 3). This fit suggests a distance modulus $(m-M)_{\mathrm{o}}=14.8 \pm 0.1$ for NGC 6496 and $(m-M)_{\mathrm{o}}=13.6 \pm 0.1$ for NGC 6352, respectively, with colour excess $E(B-V)=0.25$ for both clusters. The distance modulus of NGC 6496 is consistent with that of Richtler et al. (1994), whereas that of NGC 6352 complies with the value published by Harris (1996) for this cluster. The extinction in 
the F606W and F814W bands has been approximated following the law of Cardelli et al. (1989) and the uncertainties around the colour excess towards the two clusters is discussed in detail in Sect. 4. The CMD of NGC 6496 reveals a short, stubby horizontal branch $(\mathrm{HB})$ at $m_{814} \simeq 15.5$, a characteristic which is typical of metal rich clusters and is well reproduced by the theoretical HB track of a $0.69 M_{\odot}$ star as shown by the isochrone.

To determine the MS fiducial points, we have calculated the mean $m_{606}-m_{814}$ colour for each 0.5 mag bin along the MS, clipping all the objects lying farther than $\pm 2.5 \sigma$ from the mean colour value, and then recomputed the mean colour until the convergence was reached and a fiducial MS mean ridge line was finally defined. Tables 2 and 3 list, respectively for NGC 6496 and NGC 6352, the average MS colour and the associated standard deviation $(\sigma)$ as a function of the $m_{814}$ magnitude, together with the corrected stellar counts per bin, and the completeness fraction. The faint end of the MS of NGC 6352 is about one magnitude brighter than that of NGC 6496, due to the shorter equivalent exposure time of the NGC 6352 median averaged frames.

The MS TO of NGC 6496 lies at $m_{814} \sim 19.25$. Based on the transformations linking Johnson's photometry in the $B$ and $V$ bands to the HST photometric system (Holtzman et al. 1995), one should expect the TO to be located at $V \simeq 20.15$, i.e. the same value found by Richtler et al. (1994) in their ground-based photometry of NGC 6496. For NGC 6352 one finds, after correction for reddening and the transformation to the Johnson's system, that the TO is at $V \simeq 18.8$, in excellent agreement with the value by Fullton et al. (1995).

\section{Metallicity and reddening of NGC 6496 and NGC 6352}

Published values of the metallicity of NGC 6496 cover a wide range from $[\mathrm{Fe} / \mathrm{H}]=-0.48($ Zinn \& West 1984$)$ to $[\mathrm{Fe} / \mathrm{H}]=$ -1.05 (Friel \& Geisler 1991). This is, however, hardly surprising, since, as discussed by Richtler et al. (1994), the metallicity estimated by CMD morphological indicators is usually significantly lower than that obtained through integrated aperture photometry. (Note, however, that whilst true at the time when it was made, this statement does not apply to the comparison of the MS fiducial lines that we discuss below and which is far more reliable than any integrated property.) On the other hand, metallicity and reddening estimates are strictly connected. If we consider an increase of reddening from $E(B-V)=0.19$ to $E(B-V)=0.23$, the metallicity evaluated by Friel $\&$ Geisler (1991) would increase to $[\mathrm{Fe} / \mathrm{H}]=-0.8$, in better agreement with the estimates based on CMD features which depend on metallicity, such as the slope $S$ of the red giant branch (Hartwick 1968). If estimated through the IR maps of Schlegel et al. (1998), the reddening of NGC 6496 is $E(B-V)=0.24$.

The metallicity and reddening estimates of NGC 6352, according to all the available measurements based on different methods (Zinn \& West 1984; Gratton 1987; Geisler et al. 1991; Da Costa \& Armandroff 1991; Fullton et al. 1995; Carretta \& Gratton 1997) indicate that it is closer in Iron abundance to NGC 6496 than to 47 Tuc. Following the metallicity scale of Carretta \& Gratton (1997), the Iron abundance of NGC 6352 is
$[\mathrm{Fe} / \mathrm{H}]=-0.64$, whereas for 47 Tuc they give $[\mathrm{Fe} / \mathrm{H}]=-0.7$. All the reddening estimates are in the range $E(B-V)=$ $[0.21,0.24]$, except for the one of Schlegel et al. (1998) which, in the direction of NGC 6352, yields $E(B-V)=0.34$.

To learn more about the physical properties of the stars in these clusters, we compared the CMD of NGC 6496 and NGC 6352 with that of a reference cluster, i.e. one with known reddening, metallicity, distance and age, such as NGC 104 (47 Tuc). Further information can be derived from the features of the CMD which reflect specific stellar properties and their evolution and which can be compared with theoretical isochrones of various chemical composition and ages as calibrated through field sub-dwarfs. Figure 4 shows the fiducial MS lines of NGC 6496 (squares) and NGC 6352 (crosses) as compared with that of 47 Tuc (circles), whose photometry comes from De Marchi \& Paresce (1995b). The dashed line represents the $10 \mathrm{Gyr}$ isochrone from the models of Baraffe et al. (1998) for metal content $[\mathrm{M} / \mathrm{H}]=-0.5$ in the VEGAMAG photometric system (Isabelle Baraffe 1998, private communication). For sake of comparison, we also show the $14 \mathrm{Gyr}$ isochrone of Girardi et al. (2002) for metallicity $Z=0.008$ and $Y=0.25$. The distance modulus and extinction for NGC 6496 and NGC 6352 are those given above (respectively $(m-M)_{\mathrm{o}}=$ 14.8 and $E(B-V)=0.25,13.6$ and 0.25$)$, whereas for 47 Tuc we have adopted $(m-M)_{\mathrm{o}}=13.25$ and $E(B-V)=0.04$ from Percival et al. (2002). There is overall agreement between the models and the observed sequences for absolute magnitude brighter than $M_{814} \simeq 6.5$. The deviation of the Baraffe et al. (1998) models close to the TO is probably due to the younger age of the adopted isochrone, whilst the discrepancies between the two sets of models and between these and the data below $M_{814} \simeq 7$ most likely arise because of the uncertainties in the treatment of the opacity in the atmospheres of these high metallicity cool stars. Nevertheless, the isochrone of Baraffe et al. (1998) better approximates the observed loci of NGC 6496 and NGC 6352. We shall, therefore, adopt the corresponding M/L relation to derive a MF from the observed LF.

Interestingly, since the MS of NGC 6496 and NGC 6352 lie in the same place of the diagram and have an almost identical shape, one must conclude (see e.g. Cassisi et al. 2000), that these clusters have the same chemical composition. Indeed, it is well known that the slope of the very low mass end of the MS depends strongly on the metallicity of the stars. More importantly, however, the comparison in Fig. 4 is insensitive to errors in the distance and/or reddening, in that none of them could alter the shape of the MS but only shift it solidly in the colour-magnitude plane. On this basis, one is forced to exclude the high reddening value of $E(B-V)=0.34$, suggested by the IR dust maps of Schlegel et al. (1998), as this would imply that the metallicity of NGC 6352 is considerably higher than that of NGC 6496, at variance with the overall agreement shown in Fig. 4. However, the large value of $E(B-V)$ measured by Schlegel et al. (1998) is probably due to dust behind the cluster or some diffuse infrared background in the direction of NGC 6352.

The fiducial line of 47 Tuc (circles) is reasonably well fitted by the $[\mathrm{M} / \mathrm{H}]=-0.5$ isochrone by Baraffe et al. (1998) and is at least 0.1 mag bluer than those of the two other clusters at bright 


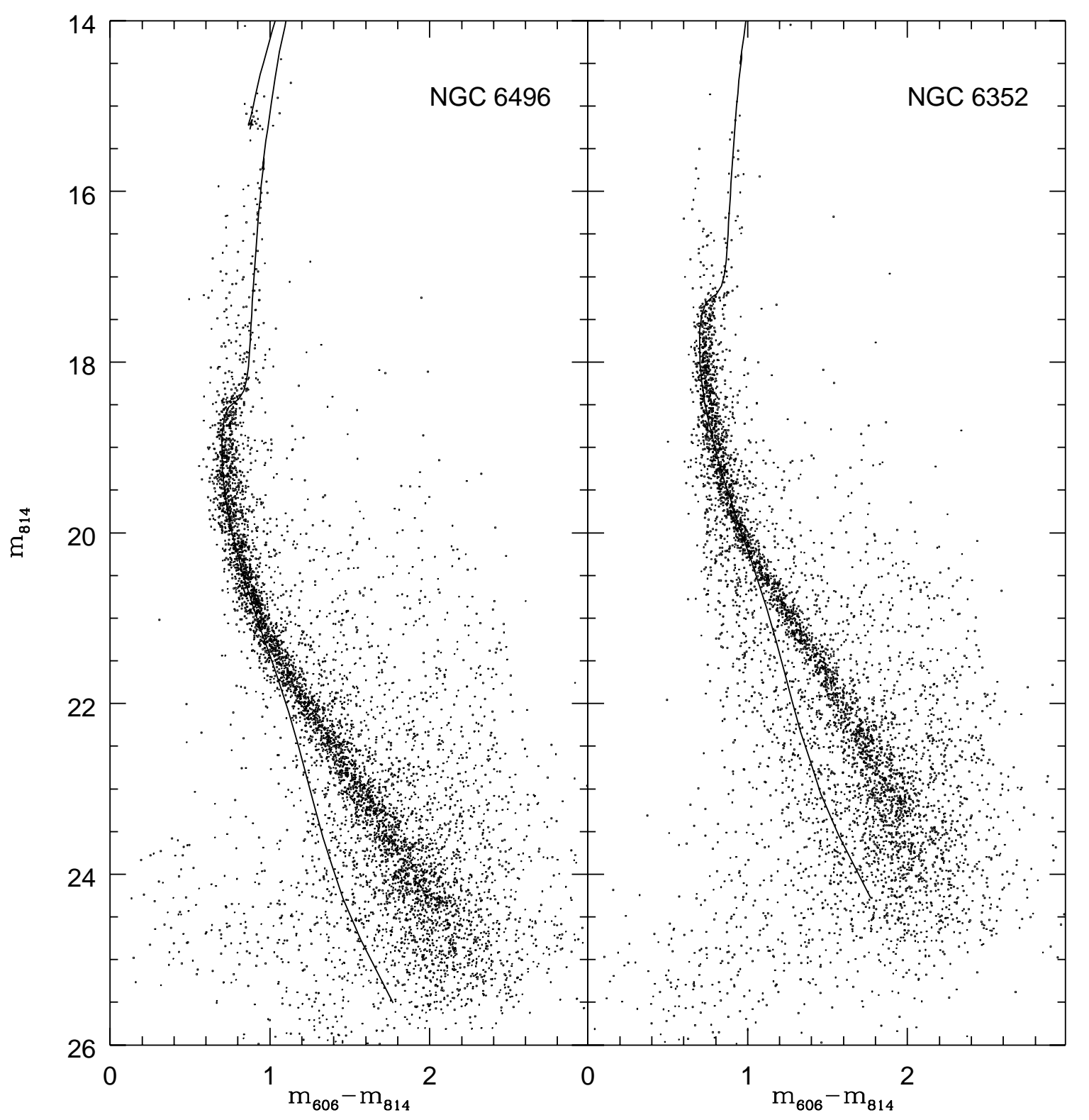

Fig. 3. CMD of NGC 6496 (left panel) and NGC 6352 (right panel). Each CMD contains all the detected objects in the short and long exposures. A 14 Gyr isochrone by Girardi et al. (2002) has been overlapped as explained in the text.

magnitudes (the difference grows larger still at lower masses) thus witnessing its lower metal content. This conclusion is further strengthened by the different slope of the MS of 47 Tuc down to $M_{814} \simeq 6$. Such an empirical estimate can be checked against the predictions of the most recent theoretical models, as we do in Fig. 5, where we compare the MF of NGC 6496 (thick solid line) and the isochrones of Baraffe et al. (1998) for a stellar population of $10 \mathrm{Gyr}$ of age. The dashed lines represent, from left to right, three $10 \mathrm{Gyr}$ isochrones of metal content, respectively, $[\mathrm{M} / \mathrm{H}]=-1,-0.5$ and and 0.0 . Only the latter (short dashed line) takes the updated $\mathrm{TiO}$ opacities into account (see below).

Firstly, we note that the $[\mathrm{M} / \mathrm{H}]=-1.0$ isochrone dose not fit at all the position nor the shape of the NGC 6496 fiducial line, so the hypothesis of an intermediate metal content for this cluster (Richtler et al. 1994) is inconsistent with the present observational and theoretical results. It is, therefore, extremely unlikely that NGC 6496 is a halo object.

The $[\mathrm{M} / \mathrm{H}]=-0.5$ isochrone, which corresponds to $[\mathrm{Fe} / \mathrm{H}]$ $\simeq-0.7$ when taking into account the overabundance of $\alpha$ elements (Ryan \& Norris 1991), fits the upper part of the MS rather well, yet it fails to reproduce the lower portion. The deviation of the models from the observed stellar locus at the bottom of the MS is a well known limitation of the theory and most probably stems from the lack of a proper treatment of the TiO molecular opacity, as extensively discussed in Baraffe et al. (1998; see also Chabrier 2001). Although a proper correction is currently not available, we show in the next section that the adoption of the theoretical $[\mathrm{M} / \mathrm{H}]=-0.5$ mass-luminosity $(\mathrm{M}-$ L) relation will still provide, in the $\mathrm{F} 814 \mathrm{~W}$ band alone, reliable results. Nevertheless, Fig. 5 allows us to conclude that the true metal content of NGC 6352 and NGC 6496 - i.e. [M/H], not 


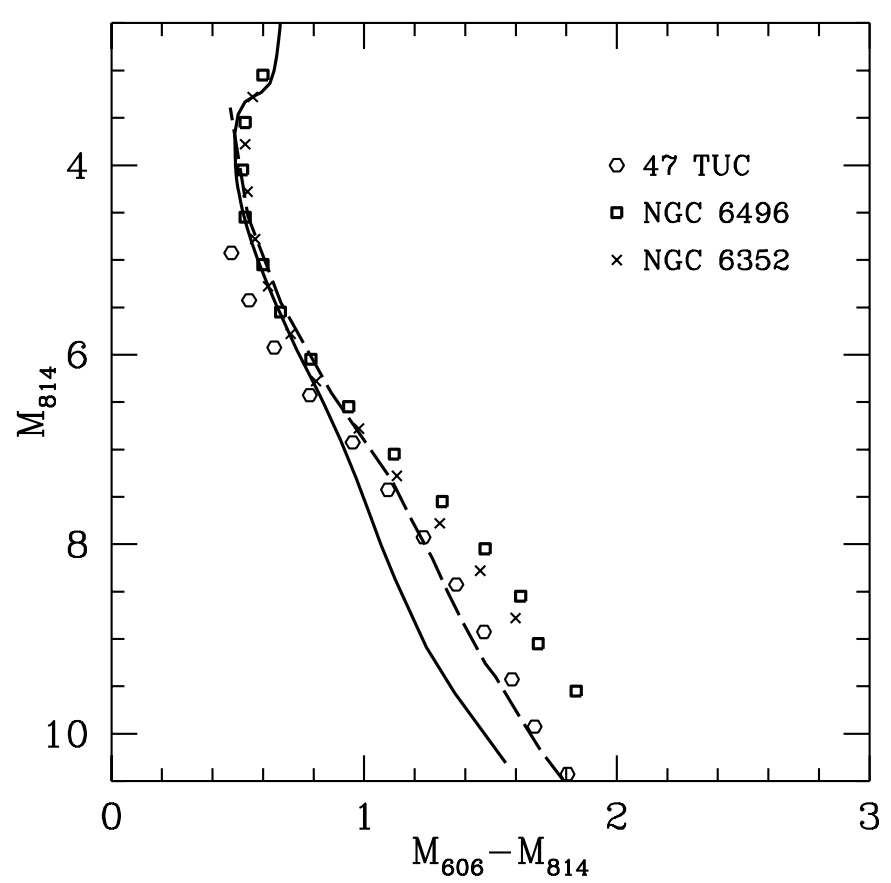

Fig. 4. Fiducial MS lines of NGC 6496 (squares) compared with that of NGC 6352 (crosses) and 47 Tuc (circles). The $14 \mathrm{Gyr}$ isochrone of Girardi et al. (2002) for $Z=0.008$ is shown (solid line) together with the $10 \mathrm{Gyr}$ isochrone by Baraffe et al. (1998) for $[\mathrm{M} / \mathrm{H}]=-0.5$ (dashed line).

just $[\mathrm{Fe} / \mathrm{H}]-$ must be at least $0.2-0.3$ dex higher than that of 47 Tuc.

\section{The luminosity and mass functions}

Tables 2 and 3 list, for each magnitude bin, the completeness fraction, the corrected LF and the corresponding rms errors coming from the Poisson statistics of the counting process (all values have been rounded off to the nearest integer). In order to derive the LF, and thus infer the PDMF of the clusters, we must correct the observed counts for the incompleteness of the sample due to crowding and to saturated stars, whose bright halo can mask possible faint objects in their vicinity. Completeness corrections have been estimated by running artificial star tests in both bands and in the combined images for each chip. For each 0.5 mag bin we have carried out 10 trials by adding a fraction of $10 \%$ of the total number of objects in that bin (see Sect. 2), and using the PSF from the co-added frames. These trials were followed by running the tasks daophot.daofind and daophot.allstar, with the same parameters used in the reduction of the scientific images to assess the fraction of the objects recovered by the procedure and the associated photometric errors.

We have divided the MS into magnitude bins, each spanning 0.5 mag from $m_{814}=18$ to $m_{814}=25.5$, and from $m_{814}=$ 17 to $m_{814}=24.5$ for NGC 6496 and NGC 6352, respectively. By adopting a sigma clipping criterion (De Marchi \& Paresce 1995b), from the CMD of Fig. 3 we have measured the LF by counting the objects in 0.5 mag bins and within \pm 2.5 times the $m_{606}-m_{814}$ colour standard deviation $(\sigma)$ around the MS ridge line. As regards the decontamination of the LF from the field

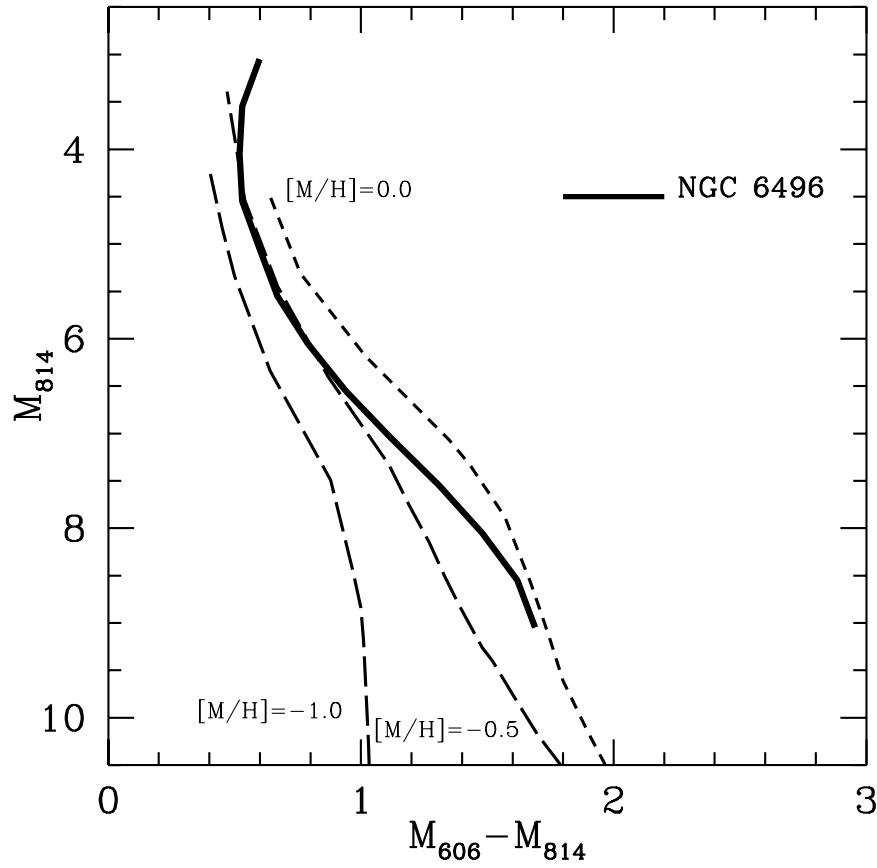

Fig. 5. Theoretical isochrones from Baraffe et al. (1998) (dashed lines) compared with the MS fiducial line of NGC 6496 (solid line).

stars, we have assumed that the field objects fill uniformly the colour range $0.3<m_{606}-m_{814}<3$. For each magnitude bin, we have estimated the number of stars with colour in this range, but outside the $5 \sigma$ wide MS belt. By dividing the measured value by the corresponding colour range and multiplying the result by the MS colour width, we have obtained the number of field stars inside the MS. The LF corrected for incompleteness and field star contamination is shown in Fig. 6, together with that of 47 Tuc, as a function of the absolute $M_{814}$ magnitude. The LF of NGC 6496 and NGC 6352 rise continuously, all the way up to $M_{814} \simeq 9$, where they reach their maximum, and drop to the detection limit at $M_{814} \simeq 10$. Taken at face value, after its peak the LF of NGC 6352 seems to decrease somewhat more steeply than that of NGC 6496, as indicated by the $1 \sigma$ error bars. We should note, however, that the difference between the two LF in practice vanishes if we consider the uncertainty brought about by our $0.5 \mathrm{mag}$ wide bins along the abscissa. Moreover, if we had assumed for the distance modulus of NGC 6496 the canonical value of $(m-M)_{I}=15.5$ as given by Harris (1996), the LF of the two clusters would have been in reasonable agreement with one another (see dashed line in Fig. 6). The value of $(m-M)_{I}=15.2$ that we have used was suggested by the remarkably good match between the MS fiducial lines of the two clusters that it would provide in the CMD (see Sect. 4). The value $(m-M)_{I}=15.5$ would imply that NGC 6496 should be slightly more metal rich than NGC 6352, a hypothesis that we cannot certainly rule out at the present level of accuracy. We, therefore, conclude that the LF of NGC 6496 and NGC 6352 are substantially the same.

To best fit the LF of the three clusters under examination, we have to fold an input MF through the derivative of the M$\mathrm{L}$ relation appropriate for the cluster metallicity. We adopted the $\mathrm{M}-\mathrm{L}$ relation obtained from the $10 \mathrm{Gyr}$ isochrones of 
Table 2. Main sequence fiducial points, luminosity function and completeness for NGC 6496.

\begin{tabular}{ccccc}
\hline \hline$m_{814}$ & $m_{606}-m_{814}$ & $\sigma$ & $N_{\mathrm{o}}$ & Compl. (\%) \\
\hline 18.25 & 0.81 & 0.06 & 80 & 100 \\
18.75 & 0.74 & 0.03 & 140 & 99 \\
19.25 & 0.73 & 0.04 & 222 & 99 \\
19.75 & 0.77 & 0.04 & 248 & 98 \\
20.25 & 0.81 & 0.05 & 288 & 97 \\
20.75 & 0.88 & 0.05 & 320 & 96 \\
21.25 & 1.00 & 0.06 & 325 & 95 \\
21.75 & 1.15 & 0.09 & 330 & 94 \\
22.25 & 1.33 & 0.09 & 356 & 91 \\
22.75 & 1.52 & 0.15 & 451 & 90 \\
23.25 & 1.69 & 0.21 & 522 & 87 \\
23.75 & 1.83 & 0.22 & 569 & 84 \\
24.25 & 1.90 & 0.21 & 636 & 80 \\
24.75 & 2.05 & 0.36 & 586 & 74 \\
25.25 & 2.16 & 0.23 & 532 & 54 \\
\hline
\end{tabular}

Table 3. Main sequence fiducial points, luminosity function and completeness for NGC 6352.

\begin{tabular}{ccccc}
\hline \hline$m_{814}$ & $m_{606}-m_{814}$ & $\sigma$ & $N_{\mathrm{o}}$ & Compl. $(\%)$ \\
\hline 17.25 & 0.77 & 0.03 & 35 & 100 \\
17.75 & 0.74 & 0.02 & 67 & 99 \\
18.25 & 0.75 & 0.03 & 95 & 98 \\
18.75 & 0.78 & 0.04 & 119 & 97 \\
19.25 & 0.83 & 0.07 & 172 & 96 \\
19.75 & 0.90 & 0.07 & 250 & 95 \\
20.25 & 1.00 & 0.11 & 267 & 93 \\
20.75 & 1.17 & 0.14 & 297 & 94 \\
21.25 & 1.33 & 0.15 & 294 & 93 \\
21.75 & 1.53 & 0.27 & 368 & 90 \\
22.25 & 1.68 & 0.27 & 447 & 87 \\
22.75 & 1.83 & 0.26 & 527 & 82 \\
23.25 & 1.87 & 0.27 & 464 & 78 \\
23.75 & 1.94 & 0.29 & 379 & 73 \\
24.25 & 1.88 & 0.42 & 353 & 60 \\
\hline
\end{tabular}

Baraffe et al. (1998) with $[\mathrm{M} / \mathrm{H}]=-0.5$ and $Y=0.25$ in the F814W filter for the reasons outlined above. When looking at the admittedly rather poor fit of the theoretical isochrones to the MS loci of these clusters in Fig. 3 below $M_{814} \simeq 7$, it is fair to wonder whether the resulting $\mathrm{M}-\mathrm{L}$ relations are reliable at all. We have, thus, examined how an improvement in the treatment of the $\mathrm{TiO}$ opacity could alter the $\mathrm{M}-\mathrm{L}$ relation and give a better fit. For the solar mixture, Baraffe et al. (1998) provide isochrones with and without TiO opacity enhancement. We obtained $\mathrm{M}-\mathrm{L}$ relations with and without this $\mathrm{TiO}$ effect and realised that the resulting MF were substantially the same, thus suggesting (although not proving) that in the case of $[\mathrm{M} / \mathrm{H}]=-0.5$ as well the adoption of the uncorrected $\mathrm{M}-\mathrm{L}$ should give reliable results. Moreover, the quoted numerical experiments show that the effects of the uncertainty of the $\mathrm{TiO}$ opacity values on the $\mathrm{M}-\mathrm{L}$ relation should be negligible when compared to the smoothing of the observed LF caused by the 0.5 mag binning.
We compare in Fig. 7 the observed LF with that obtained when a model MF is folded through the M-L relation. The squares and crosses refer, respectively, to NGC 6496 and NGC 6352. We have made here the simplifying assumption that the MF of the stellar population near the cluster halfmass radius is represented by a power-law, i.e. that the mass distribution function takes on the form $\mathrm{d} N / \mathrm{d} m \propto m^{-\alpha}$, finding that values of $\alpha=0.7 \pm 0.1$ and $0.6 \pm 0.1$ are adequate for NGC 6496 and NGC 6352, respectively. As a short-dashed line in Fig. 7 we also show the model LF that best fits the observations of $47 \mathrm{Tuc}$, the prototypical cluster of intermediate metallicity. It is well known that a simple power-law MF cannot adequately reproduce the MS LF of 47 Tuc over the whole observed range of luminosity and that a log-normal distribution in mass (Paresce \& De Marchi 2000) or a tapered powerlaw function (De Marchi et al. 2003) give a better fit over the whole mass range. Nevertheless, for the sake of the discussion that follows, it is sufficient to limit the comparison amongst the three MF within the range $0.3<m / m_{\odot}<0.8$, where a single exponent power-law is the simplest and, hence, the preferred assumption. Figure 7 leaves no doubt that the MF of 47 Tuc, with $\alpha=1.4$, is twice as steep as that of the other two clusters.

\section{Discussion and conclusions}

To the expert eye, this discrepancy would probably not come as a surprise, since it is the natural consequence of the already different LF seen in Fig. 6. To be precise, however, the difference in the LF per se would not necessarily imply that also the underlying MF should be different since 47 Tuc has a lower metal content than the other two objects. Thus, stars of equal mass would appear slightly brighter in 47 Tuc due to the different $\mathrm{M}-\mathrm{L}$ relation, therefore changing the shape of the observed LF. In fact, it is well understood that the most notable feature in the LF of a cluster, i.e. the brightness at which it peaks, shifts to fainter magnitudes with increasing metallicity (see e.g. D'Antona 1998; Kroupa \& Tout 1997; von Hippel et al. 1996; De Marchi \& Paresce 1995b) to the point that, if the distance to a cluster were known, the magnitude of the peak could be used as a metallicity indicator.

The application of the $\mathrm{M}-\mathrm{L}$ relations appropriate to each cluster has, however, removed the signature of the metallicity. As explained in Sect. 5, although some uncertainties exist as to the exact shape of the $\mathrm{M}-\mathrm{L}$ relationship in the range $-0.5<$ $[\mathrm{Fe} / \mathrm{H}]<0.0$ (Baraffe et al. 1998), they cannot be held responsible for the large discrepancy in the MF seen in Fig. 3. We must, therefore, accept that NGC 6352 and NGC 6496 have MF which are inherently different from that of 47 Tuc and from the dozen lower metallicity halo clusters studied to date in detail with the HST (Paresce \& De Marchi 2000).

The effects of energy equipartition and the resulting mass segregation have been observed and studied in detail in several clusters and can be modelled satisfactorily (47 Tuc, Paresce et al. 1994; NGC 7078, De Marchi \& Paresce 1996; NGC 6397, King et al. 1995; De Marchi et al. 2000; NGC 6121, Pulone et al. 1999; NGC 6656, Albrow et al. 2001). It is today understood that, near the cluster's half-mass radius, the local MF faithfully reflects the global MF of the whole cluster (as 


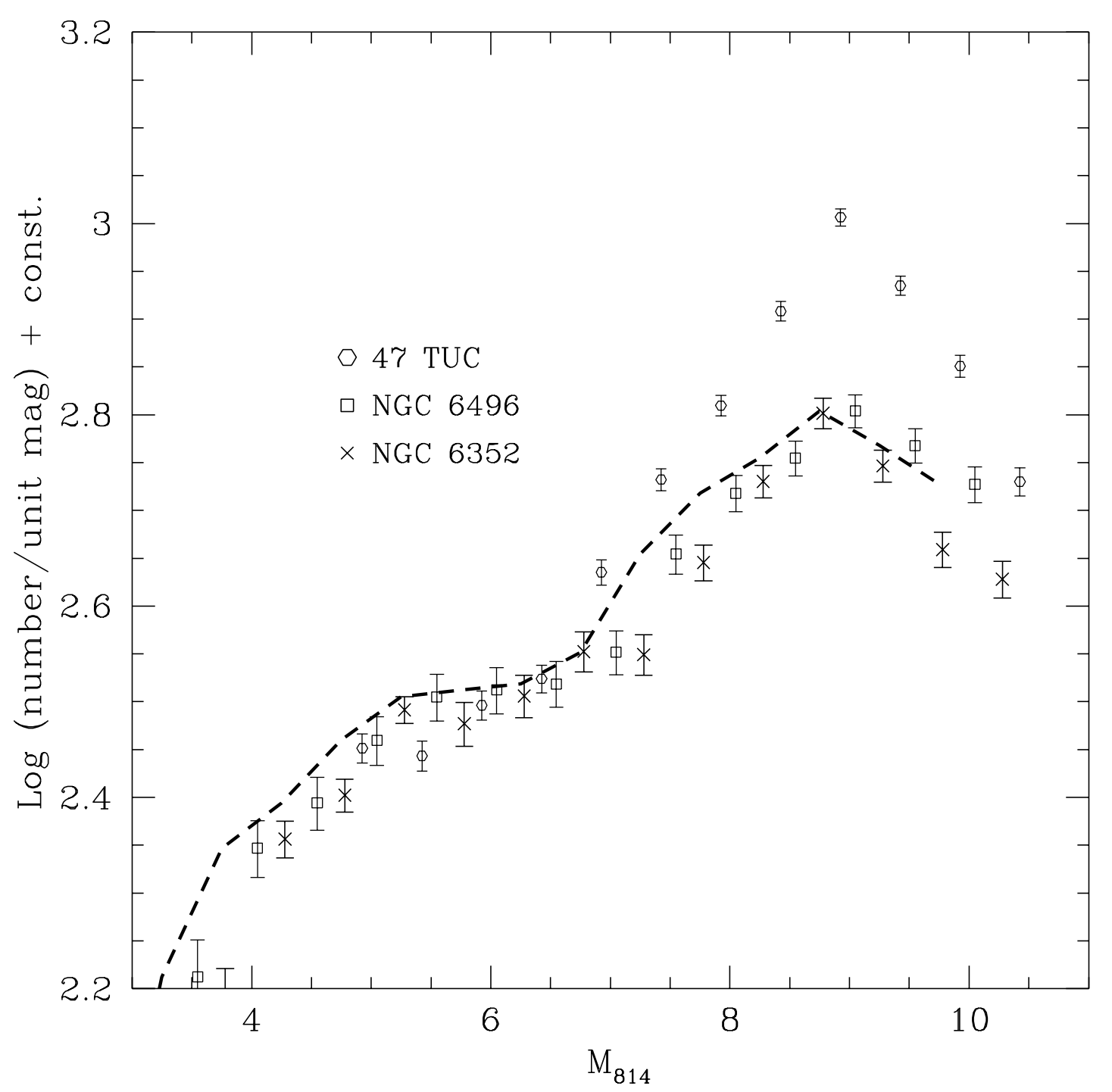

Fig. 6. Measured LF of NGC 6496 (boxes) and NGC 6352 (crosses) as compared with that of NGC 104 (circles). The dashed line shows the LF of NGC 6496 obtained by adopting the distance modulus $(m-M)_{I}=15.5$ as derived from the catalogue of Harris (1996).

originally suggested by Richer et al. 1991). Since the observations of NGC 6352 and NGC 6496 on which we here report were conducted at their half-mass radius (see Sect. 2), the effects of internal dynamics can be safely excluded from the picture.

On the other hand, the action of the Galactic tidal field can be equally strong in reshaping the MF of a cluster through disc and bulge shocks during its repeated passages at the perigalacticon. For example, the MF of NGC 6712 measured near the half-mass radius (De Marchi et al. 1999; Andreuzzi et al. 2001) bears no resemblance to that of any other cluster studied so far, in that if falls steeply with decreasing mass. Pal 5 (Grillmair \& Smith 2001) and E 3 (van den Bergh et al. 1980) might have undergone a similar, although less severe fate. It is, thus, fair to wonder whether the MF of NGC 6352 and NGC 6496 were similarly affected by the Galaxy.

Gnedin \& Ostriker (1997) and Dinescu et al. (1998) used the mass, size and space motion parameters of most Galactic globular clusters to infer the magnitude of their past dynamical interaction with the potential field of the Galaxy. They define a useful parameter, the time to disruption $\left(t_{\mathrm{d}}\right)$, which should directly correlate with the strength and extent of the overall tidal interaction and, thus, ultimately with the shape of the present day MF. However, whilst it is true that for NGC 6712 they predict a probability of disruption about two orders of magnitude higher than average, Paresce \& De Marchi (2000) have shown that no correlation exists between $t_{\mathrm{d}}$ and the shape of the MF of the 12 clusters that they studied in detail with the HST. The value of $t_{\mathrm{d}}$ must thus be used with care and we shall do so here to show, with a very simple reasoning, that neither NGC 6352 nor NGC 6496 can have been severely affected by tidal stripping. If the theory of Gnedin \& Ostriker (1997) is indeed correct, the destruction rate for NGC 6496 is about 300 times larger than that of NGC 6352, yet the two clusters have the same global MF! So, if we want these clusters to have had an IMF originally as steep as that of 47 Tuc, we cannot hold tidal stripping accountable for the alleged flattening. Moreover, postulating that the two IMF were originally different from one another but that, through dynamical evolution, they have been brought into agreement would look highly contrived, in light 


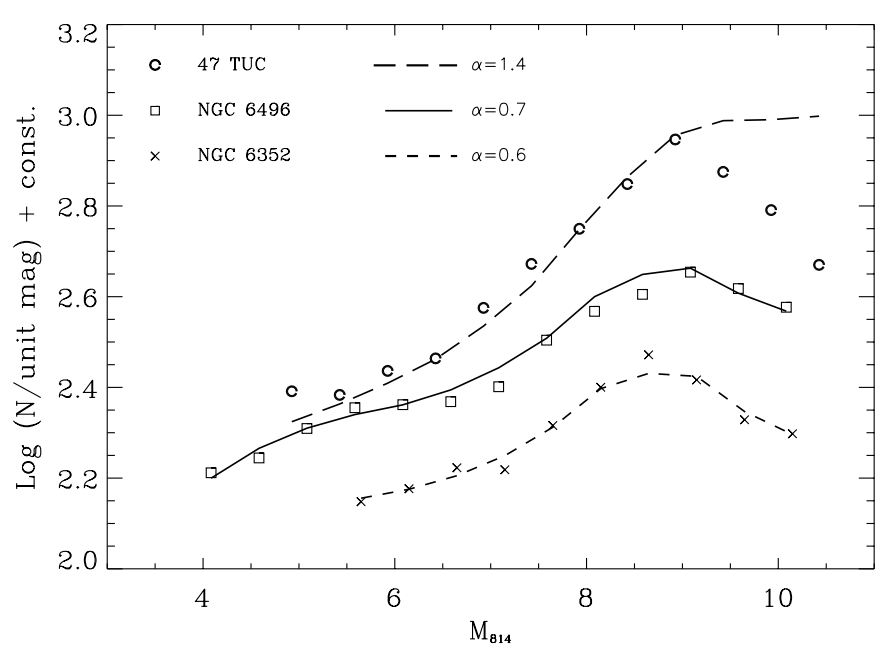

Fig. 7. Observed LF of NGC 6496 (squares), NGC 6352 (crosses), and 47 Tuc (open circles) compared with those obtained by folding a power-law MF of appropriate index through the $\mathrm{M}-\mathrm{L}$ relation of Baraffe et al. (1998) for metallicity $[\mathrm{M} / \mathrm{H}]=-0.5$.

of the widely different dynamical histories of the two clusters. Lacking any other viable explanation, it is more logical to conclude that the IMF of these clusters did not change with time, i.e. that it was already flatter at birth and the same for both.

This conclusion, however, does still not explain why the IMF of NGC 6352 and NGC 6496 should be flatter than that of the less metal rich GC unless, of course, it is the metallicity itself that governs the shape of the IMF. Claims of an apparent correlation between the shape of the MF and cluster metallicity have been put forth in the past by McClure et al. (1986), who analysed a set of nine, relatively deep GC LF obtained from the ground and noticed a shallower slope for richer clusters, in the same sense that we report here. More recent data, however, do not confirm these results (Paresce \& De Marchi 2000). Von Hippel et al. (1996) went even further and studied how metallicity affects the shape of the IMF of clusters of various ages (from young open cluster to globulars), concluding in favour of an invariant IMF.

Whether stars form through the hierarchical fragmentation of a proto-stellar cloud (Elmegreen 1999) or through the accretion of smaller clumps (Bonnell et al. 2001), once the pre-MS phase begins, the metallicity and ensuing atmospheric opacity will eventually determine the limiting mass for stable Hydrogen burning (see e.g. D’Antona \& Mazzitelli 1996) and, thus, the value of the IMF peak mass. In other words, the metal content of the natal cloud will affect its temperature and pressure, on which the characteristic mass eventually depends (Larson 1998). If turbulent fragmentation prevails below $\sim 1 M_{\odot}$, its dependence on the cooling rate and, hence, on the metallicity could affect the shape of the IMF, but no clear prediction as to the results of this process exists (Larson 2002). Moreover, the observational evidence seems overwhelming that the IMF is universal and independent of the total mass, density, age and metallicity of the stellar population (Scalo 1998; Gilmore 2001; but see also the cautionary remark in Kroupa 2001).
NGC 6352 and NGC 6496 would seem, at least apparently, to violate this rule. We have, however, so far ignored the important contribution of binaries (Kroupa 2001). Other examples exist of relatively old and metal rich systems that display a rather flat MF: the bulge (Holtzman et al. 1998; Zoccali et al. 2000), the spheroid (Gould et al. 1997) as well as the Galactic disc in the solar neighbourhood (Kroupa et al. 1993; Ried \& Gizis 1997; Gould et al. 1997). In all cases, the MF of these systems, as derived directly from the observed LF, is rather flat if no account is taken of the presence of binaries: for instance, for stars less massive than $\sim 0.5 M_{\odot}$ Gould et al. (1997) find $\alpha=0.56$ in the disc and $\alpha=0.75$ in the spheroid. Ignoring the binaries, however, results in the underestimate of the real IMF slope by an amount that depends on the slope itself and on the binary fraction (Sagar \& Richtler 1991; Kroupa 2001) and which can be of order $\Delta \alpha \simeq 0.5$ or higher. As Holtzman et al. (1998) show, a measured MF slope of $\alpha \simeq 0.7$ can be in reality as steep as $\alpha \simeq 1.3$ if the binary fraction is of order $50 \%$.

The age and metallicity of the stellar population in NGC 6496 and NGC 6352 should be rather similar to those of the stars in the bulge, as one can judge for instance from the similarity of their CMD and particularly of their curved red giant branch and clumped horizontal branch (Richtler et al. 1994; Fullton et al. 1995; Zoccali et al. 2000). It is, then, reasonable to apply to their MF a correction similar to that derived by Holtzman et al. (1998), which would in turn bring them into good agreement with that of 47 Tuc and of the other, lower metallicity halo clusters (Paresce \& De Marchi 2000).

At this juncture, one might wonder whether a similar correction for binaries should be applied to all GC, including 47 Tuc. Albrow et al. (2001), however, have recently conducted high precision differential time series photometry on $\sim 46000$ stars in the core of this cluster and have concluded that the binary fraction is on average $\sim 13 \%$ within $\sim 4$ core radii $\left(r_{\mathrm{c}}\right)$, with indications that it drops to $\sim 8 \%$ or less outside of $2.5 r_{\mathrm{c}}$. A correction to the IMF in this case is, therefore, not necessary. The only notable signature of binaries on the observed CMD is a vertical broadening of the MS (of up to 0.75 mag along the magnitude axis for equal mass systems), which should be best observed where the MS slope is not too steep in the CMD, or where it changes abruptly near the MS kinks due to changes in the main source of opacity (see, e.g., the CMD of NGC 6752 by Rubenstein \& Bailyn 1997). With the advent of high precision HST photometry, this test has become possible (De Marchi \& Paresce 1995b; Elson et al. 1995; Cool et al. 1996) and has revealed the paucity of these objects. Yet, in only a few cases, for example NGC 6752 (Rubenstein $\&$ Bailyn 1997), has the photometry been sufficiently precise to detect this binary sequence.

The MS of NGC 6496 and NGC 6352 is, however, much steeper than that of NGC 6752 in the magnitude range where the photometric uncertainty would allow us to see the effects of a broadening. Below $m_{606} \simeq 23$ the MS of NGC 6496 flattens out, but here the photometric uncertainty dominates (see Table 2). Furthermore, since the vertical displacement of a binary with respect to the MS in the CMD depends on the mass ratio $q=m_{2} / m_{1}$, it will be easier to detect nearly equal mass systems, which are however believed to be a small fraction of 
the total, at least according to Duquennoy \& Mayor (1991). These authors investigate the multiplicity of nearby $\mathrm{G}$ dwarfs and find that the secondary mass distribution peaks at around $q \simeq 0.25$, where the solar neighbourhood's IMF has its maximum (Kroupa et al. 1993), thereby suggesting that binaries should form some time after the formation of single stars. If the same mass distribution applies to the binaries in NGC 6496 and NGC 6352, their signature on the MS will remain undetected, at least at the present level of photometric accuracy, since a typical $\sim 0.25 M_{\odot}$ companion would add insignificantly to the brightness of a more massive primary. Although for late $\mathrm{M}$ dwarf primaries the $q$ distribution seems somewhat flatter than for G stars (Fischer \& Marcy 1992), again in agreement with a binary formation scenario through random sampling from the same IMF, our photometric uncertainty, the contamination due to field objects and the steepening of the MS in the CMD plane prevent us from reliably probing this region of the parameter space.

Whilst NGC 6352 and NGC 6496 are the only two clusters in the bulge for which a deep MF has been measured so far, at this stage one could tentatively conclude that the main effect of the metallicity difference between these and the halo clusters may indeed be the binary frequency. The proposed universality of the IMF would not seem to be affected by this conclusion, but it must not be disconnected from the process of binary formation. In order for the final stellar IMF to be the same regardless of the metal content, it would require that stars are all born single and that only later they aggregate in multiple systems within the natal cloud (where they are observed), in a way which however depends on the metallicity. That the properties of binaries can be better explained through a "two step" IMF has been discussed recently by Durisen et al. (2001). Thus, it cannot be excluded, for instance, that the stronger UV flux and more powerful winds of OB stars in metal poor clusters would not only hinder the formation of low mass objects (Larson 1998), but also discourage their interaction and aggregation into multiple systems. This scenario agrees with the finding (Duquennoy \& Mayor 1991; Fischer \& Marcy 1992) that, in the solar neighbourhood, binary formation seems to happen through random association of two stars drawn from the same IMF. An alternative proposal is also possible in which, after the initial collapse, clumps form which further fragment into single stars or multiple systems depending on the cloud's metallicity. This hypothesis would, however, be less favoured because in order to conserve the uniformity of the stellar IMF with metallicity the fragmentation mechanism should adapt itself to the metal content in a way which is presently not understood.

In spite of the rather speculative nature of the previous paragraph, we stress here that our result is the first tentative indication of a large fraction of binaries in some globular clusters.

\section{Summary}

The main results of this paper can be summarised as follows:

1. Two regions located at the respective half-light radius of NGC 6496 and NGC 6352 were observed with the WFPC2 on board the HST. The photometry of the WF chips, carried out in the F814W and F606W bands, extends down to $m_{814} \sim 25.5$ corresponding to $M_{814} \simeq 10.5$ with completeness in the raw data always above $\sim 50 \%$.

2. The MS fiducial lines of NGC 6496 and NGC 6352 show a similar shape within the measurement errors, thus strongly suggesting a similar metal content, which appears at least $0.2-0.3$ dex higher in $[\mathrm{M} / \mathrm{H}]$ than that of 47 Tuc. Accordingly, neither object should be considered a member of the halo Galactic globular cluster system.

3. No theoretical M-L relationships are available that reproduce the shape of the MS fiducial line over the whole range of luminosity spanned by the observations. According to Baraffe et al. (1998), the offset observed at low luminosities (and masses) is a shortcoming reflecting the inadequate treatment of the $\mathrm{TiO}$ molecule, which becomes important for metallicity higher than $[\mathrm{M} / \mathrm{H}] \simeq-0.5$. This effect is less severe in the reddest bands.

4. The $I$-band LF of NGC 6496 and NGC 6352, obtained from the CMD using both the colour and magnitude information and corrected for incompleteness were compared to the LF of 47 Tuc. Although all three LF feature a peak at a similar magnitude level $\left(M_{814} \simeq 9\right)$, that of 47 Tuc rises more steeply to the maximum.

5. Using the M-L of Baraffe et al. (1998), the power-law MF that best fit the observations in the range $0.3<\mathrm{m} / \mathrm{m}_{\odot}<0.8$ are derived and compared, finding an index $\alpha=0.7$ and 0.6 for NGC 6496 and NGC 6352, respectively. The MF of 47 Tuc is twice as steep over the same mass range, with $\alpha=1.4$ and similarly steep is the MF of other halo GC.

6. We investigate the origin of this discrepancy and conclude that a dynamical origin for it is highly unlikely if present theories are correct. We suggest instead that it could be the result of a large binary fraction amongst the stars of NGC 6496 and NGC 6352. A binary fraction of $\sim 50 \%$ would produce a stellar MF in agreement with that of 47 Tuc and of the low metallicity halo clusters. Because of their location and chemical properties, both NGC 6496 and NGC 6352 are likely members of the bulge, where such a binary fraction is accepted (Holtzman et al. 1998). If this result is confirmed, it would imply that the metallicity affects the way in which binaries form and aggregate, but not the fragmentation process of the natal cloud. A larger sample of intermediate and high metallicity GC is needed to address these issues in more detail.

Acknowledgements. It is our pleasure to thank I. Baraffe for useful discussions. L. Pulone is grateful to ESO, where he conducted part of this work, for the hospitality of their visitor programme. We are very thankful to an anonymous referee whose comments have considerably improved the presentation of this paper. This work has been partly supported by MURST/COFIN 2000 under the project: "Stellar observables of cosmological relevance."

\section{References}

Albrow, M., Gilliland, R., Brown, T., et al. 2001, ApJ, 559, 1060

Andreuzzi, G., De Marchi, G., Ferraro, F. R., et al. 2001, A\&A, 372, 851 
Armandroff, T., \& Zinn, R. 1988, AJ, 96, 92

Armandroff, T. 1988, AJ, 96, 588

Armandroff, T. 1989, AJ, 97, 375

Baraffe, I., Chabrier, G., Allard, F., \& Hauschildt, P. 1998, A\&A, 337, 403

Bonnell, I., Clarke, C., Bate, M., \& Pringle, J. 2001, MNRAS, 324, 573

Burnstein, D., \& Heiles, C. 1982, AJ, 87, 1165

Carretta, E., \& Gratton, R. 1997, A\&AS, 121, 95

Cassisi, S., Castellani, V., Ciarcelluti, P., Piotto, G., \& Zoccali, M. 2000, MNRAS, 315, 679

Cardelli, J. A., Clayton, G. C., \& Mathis, J. S. 1989, ApJ, 345, 245

Chabrier, G. 2001, ApJ, 554, 1274

Cool, A., Piotto, G., \& King, I. 1996, ApJ, 468, 655

Copeland, H., Jensen, J., \& Jorgensen, H. E. 1970, A\&A, 5, 12

D'Antona, F. 1995, in The bottom of the Main Sequence - and beyond, ESO Astrophysics Symposia (Springer), ed. C. Tinney, 13

D'Antona, F. 1998, in The stellar initial mass function, ASP Conf. Ser., 142, ed. G. Gilmore, \& D. Howell (San Francisco: ASP), 157

D’Antona, F., \& Mazzitelli, I. 1996, ApJ, 456, 329

De Marchi, G., Leibundgut, B., Paresce, F., \& Pulone, L. 1999, A\&A, 343, 9L

De Marchi, G., \& Paresce, F. 1995a, A\&A, 304, 202

De Marchi, G., \& Paresce, F. 1995b, A\&A, 304, 211

De Marchi, G., \& Paresce, F. 1996, ApJ, 467, 658

De Marchi, G., Paresce, F., \& Portegies Zwart, S. 2003, ApJ, in preparation

De Marchi, G., Paresce, F., \& Pulone, L. 2000, ApJ, 530, 342

Dinescu, D., Girard, T., van Altena, W., Mendez, R., \& Lopez, C. 1997, AJ, 114, 1014

Druisen, R., Sterzik, M., \& Pickett, B. 2001, A\&A, 371, 952

Duquennoy, A., \& Mayor, M. 1991, A\&A, 248, 485

Elmegreen, B. 1999, ApJ, 515, 323

Elson, R., Gilmore, G., Santiago, B., \& Casertano, S. 1995, AJ, 110, 682

Fischer, D., \& Marcy, G. 1992, AJ, 396, 178

Friel, E., \& Geisler, D. 1991, AJ, 101, 1338

Fullton, L., Carney, B., Olszewski, E., et al. 1995, AJ, 110, 652

Gilmore, G. 2001, in Starburst galaxies near and far, ed. L. Tacconi, \& D. Lutz (Heidelberg: Springer-Verlag), 34

Girardi, L., Bertelli, G., Bressan, A., et al. 2002, A\&A, 391, 195
Gould, A., Bahcall, J., \& Flynn, C. 1997, ApJ, 482, 913

Gnedin, O., \& Ostriker, J. 1997, ApJ, 474, 223

Grillmair, C., \& Smith, G. 2001, AJ, 122, 3231

Harris, W. 1996, AJ, 112, 1487

Hartwick, F. 1968, ApJ, 154, 475

Holtzman, J., Burrows, C., Casertano, S., et al. 1995, PASP, 107, 1065

Holtzman, J., Watson, A., Baum, W., et al. 1998, AJ, 115, 1946

King, I., Sosin, C., \& Cool, A. 1995, ApJ, 452, L33

Kroupa, P. 2001, MNRAS, 322, 231

Kroupa, P., \& Tout, C. 1997, MNRAS, 287, 402

Kroupa, P., Tout, C., \& Gilmore, G. 1993, MNRAS, 263, 545

Larson, R. 1998, MNRAS, 301, 569

Larson, R. 2002, in Galactic star formation across the stellar mass spectrum, ed. J. de Buizer (San Francisco: ASP), in press [astro-ph/0205466]

McCLure, R., Vandenberg, D., Smith, G., et al. 1986, ApJ, 307, L49

Meylan, G., \& Heggie, D. 1997, A\&ARv, 8, 1

Paresce, F., \& De Marchi, G. 2000, ApJ, 534, 870

Paresce, F., De Marchi, G., \& Jedrzejewski, R. 1995, ApJ, 442, L57

Percival, S., Salaris, M., van Wyk, F., \& Kilkenny, D. 2002, ApJ, 573, 174

Pulone, L., De Marchi, G., \& Paresce, F. 1999, A\&A, 342, 440

Reid, I., \& Gizis, J. 1997, AJ, 113, 2246

Richer, H., Fahlman, G., Buonanno, R., et al. 1991, 381, 147

Richtler, T., Grebel, E., \& Seggewiss, W. 1994, A\&A, 290, 412

Rubenstein, E., \& Bailyn, C. 1997, ApJ, 474, 701

Ryan, S., \& Norris, J. 1991, AJ, 101, 1865

Sagar, R., \& Richtler, T. 1991, A\&A, 250324

Sarajedini, A., \& Norris, J. 1994, ApJS, 93, 161

Spitzer, L. 1987, Dynamical evolution of globular clusters (Princeton: Princeton University Press)

Scalo, J. 1998, in The stellar initial mass function, ed. G. Gilmore, \& D. Howell (San Francisco: ASP), ASP Conf. Ser., 142, 201

Schlegel, E., Finkbeiner, D., \& Davis, M. 1998, 500, 525

Stetson, P. 1987, PASP, 99, 191

van den Bergh, S., Demers, S., \& Kunker, W. 1980, ApJ, 239, 112

Vesperini, E., \& Heggie, D. 1997, MNRAS, 289, 898

von Hippel, T., Gilmore, G., Tanvir, N., Robinson, D., \& Jones, D. 1996, AJ, 112, 192

Zinn, R., \& West, M. 1984, ApJS, 55, 45

Zinn, R. 1985, ApJ, 293, 424

Zoccali, M., Cassisi, S., Frogel, J., et al. 2000, ApJ, 530, 418 